\title{
A passive Barkhausen noise sensor for low-power applications
}

\author{
Jesper Hamfelt*, Jonas Gustafsson, Jan van Deventer, Torbjörn Löfqvist, \\ Fredrik Häggström* and Jerker Delsing \\ *SKF University Technology Centre \\ Division of EISLAB \\ Luleå University of Technology \\ 97187 Luleå \\ Sweden \\ Email: jesper.hamfelt@ltu.se
}

\begin{abstract}
This paper proposes a passive Barkhausen noise sensor design suitable for low power applications. The sensor uses a permanent magnet and the relative motion between itself and a measured specimen instead of the conventional method that uses a fixed sensor and an alternating magnetic field. Since this novel design is passive, the sensor is well suited for low power applications and could potentially be used in e.g. a condition monitoring system integrated into a rolling element bearing. Proof of concept testing has been performed showing that the proposed sensor produces similar results as conventional Barkhausen noise sensors when applied to specimens being cyclically loaded until failure in a rotating bending rig. The results imply that material fatigue detection using the Barkhausen noise can be performed with the proposed sensor at a fraction of the energy cost compared to a conventional sensor. This warrants future research into the development of the proposed sensor, its advantages, disadvantages, and functionality.
\end{abstract}

\section{INTRODUCTION}

Today, a growing area of interest in a variety of applications is condition based maintenance (CBM) [1]. CBM is a method of performing maintenance and assuring the function of machines and components using condition monitoring (CM) [2]. The use of CBM makes it possible to perform maintenance or preventive actions before the machine or component fails, instead of either performing maintenance after the failure have already occurred, also known as unplanned maintenance or run-to-failure maintenance, or perform maintenance at periodic time intervals, regardless of the condition of the component or machine, known as time-based preventive maintenance or planned maintenance. The use of condition based maintenance prolongs the life of the machine and its components and additionally reduces the environmental effects of unneeded maintenance and replacement of healthy and working components. An enabler for CM is non-destructive testing (NDT) [3].

Most of the CM and NDT methods used today are energy and space consuming, and are therefore not suitable to be miniaturized and integrated within, for example, a rolling element bearing to monitor the rolling elements or the raceways. Acoustic emission (AE) is one technique that is well suited since it uses acoustic waves and the sensing element can be placed adjacent to the raceways or rolling elements. Vibration analysis is also usable since it measures the vibrations of the bearing and does not need to be near the source of a defect. Al-Ghamd and Mba [4] presented a comparison between AE and vibration analysis used for the identification of bearing defects. They show that both methods can detect defects of varying sizes but $\mathrm{AE}$ is found to determine defects at an earlier stage as well as more accurate estimates of the sizes of the defects. There is also ongoing work with a new lowpower eddy current testing method for the detection of cracks and subsurface defects, using a permanent magnet and a coil oriented parallel to the surface [5]. This solution could be used for integration purposes. As an NDT method, the Barkhausen noise method has been shown to be useful for the evaluation of residual stress in bearings before operations and during maintenance services [6]. This method, however, relies on a significant amount of energy to power the excitation signal and is therefore not suitable for integration in a bearing.

To make CM and NDT possible in hard to reach and/or enclosed locations, where the only available power is either harvested on location or provided by battery, low power sensor systems are needed. The sensor proposed and evaluated in this paper is well suited for low power applications since it is by its design passive and thus only needs power for the peripherals, such as signal amplification, analysis and communications. The only required conditions for the sensor to operate are that it is in relative movement to the interrogated material and that the material is ferromagnetic. The sensor consists of a permanent magnet and a solenoid coil, where the magnet is used to excite Barkhausen noise in the material and the solenoid coil to pick up the Barkhausen noise.

\section{A. Barkhausen Noise sensing}

The Barkhausen noise is a magnetic phenomenon appearing when a ferromagnetic material is magnetized [7] and it is well known that the Barkhausen noise on a microscopical level is affected by dislocations, cracks, impurities or other defects.

A conventional, or active Barkhausen noise sensor, is shown in Fig. 1. This type of sensor is typically used in NDT to detect structural changes in a specimen caused by, for example, stress [8] or fatigue [9]. To produce the Barkhausen noise, an 


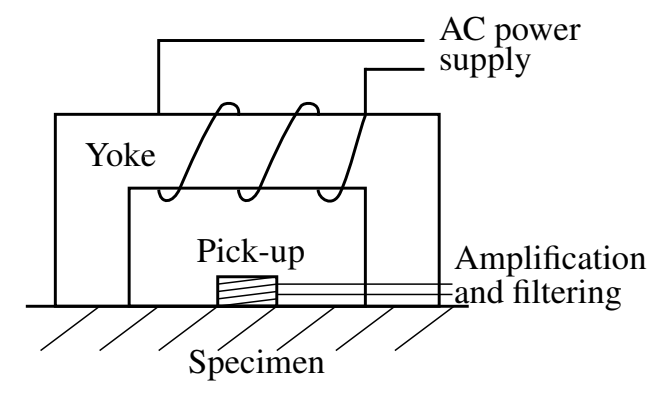

Fig. 1. Schematic figure of the conventional Barkhausen noise sensor.

alternating current is applied in a coil wound around a yoke, hereafter referred to as the excitation coil, thus inducing an alternating magnetic field in the yoke and specimen which is inductively captured by a pick-up coil. The signal sensed by the pick-up coil consists of the Barkhausen noise superimposed on the AC excitation signal and therefore the sensed signal needs to be high-pass filtered in order to attenuate the induced field from the AC excitation. The sensed signal is also low-pass filtered in order to avoid aliasing during digitisation and to suppress high-frequency background noise [10].

The use of an alternating field to create the Barkhausen noise limits the penetration depth due to the skin effect [11]. Since the signal has to be high-pass filtered, in order to remove the induced field from the AC power supply, the signals arising from deep in the material are more attenuated than the near surface signals. A few other drawbacks with a conventional sensor are that it is space consuming, especially the yoke, it has to be connected by wires and it is energy consuming, see examples in section $\mathrm{V}$. These attributes makes a conventional sensor unsuitable for CM applications in enclosed locations and limited in power.

The proposed sensor design approaches the Barkhausen noise excitation problem from an application specific perspective, where the changes in the magnetic field are due to the relative motion of a permanent magnet with respect to the specimen. This offers a solution without the power consuming electromagnetic excitation. With a pick up coil near or around the magnet, the sensor resembles a reluctance sensor with a non varying reluctance circuit. The measured signal is then the Barkhausen noise generated by the permanent magnet and the relative movement between the sensor and the specimen.

A passive Barkhausen sensor significantly reduces the power consumption by removing the need for magnetic field excitation but brings new problems and challenges to deal with, challenges which are not dealt with it this paper, but outlined in section V. The current work first focuses on providing the proof of concept that the sensor can be used to detect a change as a specimen is cyclically loaded and eventually breaks.

\section{PROPOSED BARKHAUSEN NOISE SENSOR}

The proposed sensor is a low-power solution that could be made small enough to be integrated into a bearing. The sensor is passive and requires power only to amplify the signal and, if used in a wireless system, to communicate. The passive design enables the usage of energy harvesting technologies to supply the power and can thus make it possible to provide real time condition monitoring of, e.g., bearing raceways. Additional usages of the sensor could be to provide detection of unfavourable working conditions of components and to assess component quality during and/or after production.

\section{A. Design and operation principles}

The sensor consists of at least one solenoid coil and at least one permanent magnet, arranged such that the magnetic flux from the magnet is parallel to the core of the coil, as shown in Fig. 2. This passive excitation of the Barkhausen noise removes the need for an alternating excitation field and the subsequent signal filtering. The high-pass filtering can then be moved from the range of $\mathrm{kHz}$ to only a high-pass filtering in the range of hundreds of $\mathrm{Hz}$ to remove the power line frequency noise $(50$ or $60 \mathrm{~Hz})$ and its harmonics. This reduction of the high-pass filtering frequency should allow for a deeper measurement in the material, due to deeper penetration of a low frequent signal [11]. As with the conventional sensor, a low-pass filter is necessary to remove high-frequency background noise and prevent aliasing during digitization.

When the sensor sweeps over a ferromagnetic material, the magnetic field from the permanent magnet acts on the magnetic domains in the material, making them jump in position due to magnetic hysteresis as they try align themselves to the direction of the field. This realignment creates the Barkhausen noise. The amount of induced noise dependents on the strength of the magnetic field (more domains are moved and reached with a stronger field), the distance between the sensor and the material (more of the field is allowed to act on the material as the sensor is closer) and the relative speed between the sensor and the material (higher speed gives rise to eddy currents, and thus decreases the penetration depth, however, a larger surface area is affected per time unit). The rotation of the specimen and the ellipsoidal shape of the field

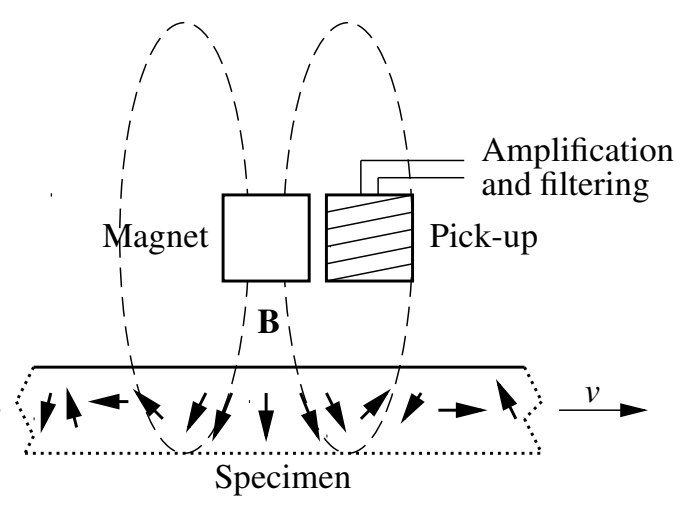

Fig. 2. Schematic figure of the proposed passive sensor scanning over a surface moving at a velocity $v$. The ellipsoid lines represent the magnetic field $\mathbf{B}$ from the permanent magnet, and the arrows in the specimen represent the orientation of the magnetic domains. 
(as illustrated in Fig. 2) prevents all the magnetic domains in the specimen to become fully aligned in a uniform direction and by that cease to give rise to Barkhausen noise.

\section{EXPERIMENTAL VERIFICATION OF THE PROPOSED BARKHAUSEN NOISE SENSOR}

Using measured values from an active Barkhausen sensor the expected Barkhausen noise from a passive sensor could be of the order $1-500 \mu \mathrm{V}$, exponentially decreasing in frequency. Thus, the design of the passive Barkhausen sensor involves a high-gain amplification circuit with a wide band-pass filter. For the presented results, the sensor configuration retained had one solenoid coil and three stacked neodymium (type N50) magnets. Each magnet had a B-field strength of $1.4 \mathrm{~T}$. The magnets were stacked in series to match the height of the solenoid coil, which was $15 \mathrm{~mm}$. The coil was made up of a $0.1 \mathrm{~mm}$ diameter copper wire wound with 1500 turns, with an air core of $2 \mathrm{~mm}$. The produced sensor prototype can be seen in Fig. 3. The prototype amplification circuit used in this work is depicted in Fig. 4. It consists of two identical inverting amplifier stages, with bandpass filtering at each stage, and a final filtering step. The total amplification of the circuit was set to $10000 \mathrm{~V} / \mathrm{V}$. The lower cut-off frequency was chosen to $250 \mathrm{~Hz}$ and the higher to $10 \mathrm{kHz}$. The value of the higher cutoff was intended to be $1 \mathrm{MHz}$ (to avoid aliasing when sampling at the maximum frequency of the data acquisition, $2 \mathrm{MHz}$ ) but was forced to be set much lower due to an external noise at $10 \mathrm{kHz}$ that interfered with the measurements.

The amplified signal was acquired using a Measurement Computing Corporation digitizer DAQ-1602HS. The setup and operation of the data acquisition was done via Mathworks's MATLAB. The amplified and filtered signal was recorded using the DAQ-1602HS with a sampling frequency of $2 \mathrm{MHz}$ and a selected input range of $[-10 ; 10]$ Volts. The signal was sampled for 1 second and paused repeatedly, the pause time differed between the test was either 1 or 5 seconds. The increase in pause time was made due to the small change of signal between each consequent measurement with a 1 second pause. This intermittent sampling was continued until each specimen failed. During each of the sampling intervals, $2 \cdot 10^{6}$ samples were recorded over 50 revolutions of the specimen. The analysis of the acquired data was performed on a personal computer using MATLAB.

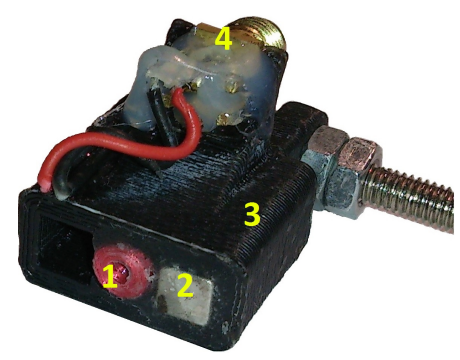

Fig. 3. The sensor prototype, showing pick-up coil (1), magnet (2), casing (3) and SMA connector (4).

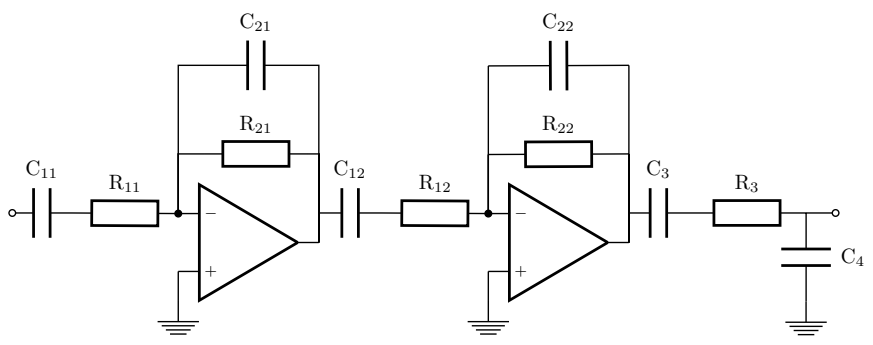

Fig. 4. Schematic of the amplifying and filtering circuit

The tests were performed on cylindrical steel rods mounted in a rotating bending rig, cf. Fig. 5. The rig continuously bent the samples as they were being rotated, and thus fatigued the rod around its circumference. For these tests the samples were fatigued in the rig until the point of breakage. The revolution speed of the test rig was constant for all tests, $3000 \mathrm{rpm}$, and the applied torque was constant at $50 \mathrm{Nm}$ through the tests. The Barkhausen noise measurements were done with the sensor placed perpendicular to the axis of the specimen and the magnet was before the pick-up coil in the rotational direction of each specimen. The distance between the sensor and the specimen was $1 \mathrm{~mm}$. Note that the tests were performed on previously fatigued specimens, and the presented results therefore do not reveal a change from a healthy specimen to failure, but a change from a state of unknown residual fatigue to failure. There were 10 specimens used in these measurements. Two of those measurements were performed with the presented sensor configuration and the results from the others are therefore not presented in this paper. All specimens had the same dimensions (cf. Fig. 5): the rods had a total length of $225 \mathrm{~mm}$, a diameter of $12 \mathrm{~mm}$, a waist with a diameter of $7 \mathrm{~mm}$, and a curvature radius of $127 \mathrm{~mm}$ for the waist.

The literature show Barkhausen noise related experiments being performed on specimens that were fatigued in the similar type of test rigs. Examples include works by Palma et al. [12] and Soultan et al. [13]. Palma et al. used the conventional Barkhausen sensor to measure on the specimens. Their measurements were performed after removing the specimens from the rig during the tests, and thus attaining information about the residual fatigue in the specimen over time as they were

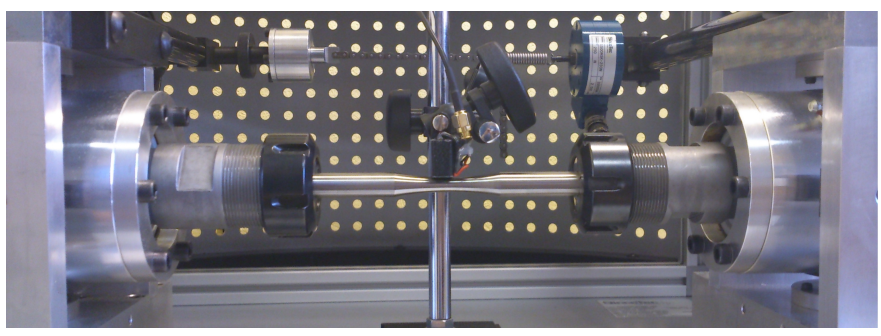

Fig. 5. Photograph of the experimental setup, showing rotating bending test rig with the sensor mounted inside. The torque applied with the chain between the levers bends the specimen as it is rotated, and thus the specimen is fatigued evenly along its circumference. 
being fatigued. Soultan et al. used no external excitation to generate the Barkhausen noise. They used only a coil wound around the specimen while it was mounted in the test rig, in doing so measured the mechanical Barkhausen noise [14]. A magnet was not necessary as the cyclic mechanical straining directly affect the magnetic domains and associated sudden magnetic realignment. These above cited papers shows that the Barkhausen noise, either mechanical or magnetic, changes as the specimen is fatigued. For the mechanical Barkhausen noise the change usually follows a specific three stage pattern: the signal amplitude increases in the beginning to stabilize on a long plateau and then decreases until failure occurs. The magnetic Barkhausen noise follows one of two patterns: either it increases rapidly in the early stage to then decrease rapidly and finally plateau for the rest of the test duration, or it increases rapidly to then slightly increase for the remainder of the test.

\section{Results}

The measured signals were analysed by calculating the RMS voltage of each measurement series and sequenced over time to obtain a timeline. This is a similar method to other works [3], [8], [10], [13]. These time lines are shown in Fig. 6, were the signal from specimen 1 is sampled with a 1 second interval and the signal from specimen 2 with a 5 second interval. The time lines were further filtered using an 11 point moving average filter as shown by the thicker line. As can be seen in the figure, the behaviour of the signals are similar between the two specimens. Furthermore the signal strength is similar, where specimen 2 produces slightly higher values. Interestingly, the signal from specimen 2 also poses much higher signal variation than seen in the specimen 1 signal. This difference could be attributed to specimen differences or an introduced external noise.

To enhance the differences between RMS voltages from the two different measurements the values were normalised and plotted together. The normalised results can be seen in Fig. 7. Comparing the results to each other, it can be seen that for both measurements the values increases sharply in the beginning to reach a maximum and then start to decrease for the remaining duration of the fatiguing. The relative change in amplitude lies in similar ranges for both measurements.

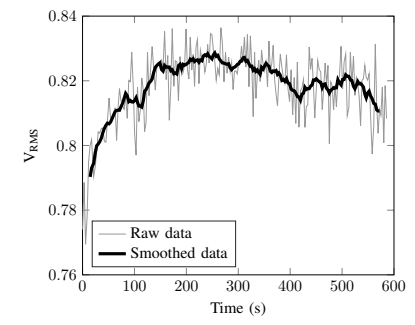

(a) Specimen 1

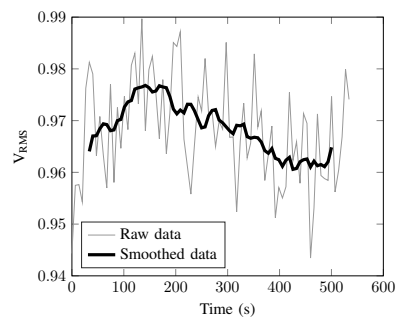

(b) Specimen 2
Fig. 6. RMS voltages from measurements on two specimens applied with a $50 \mathrm{Nm}$ torque and rotated at $3000 \mathrm{rpm}$ until breakage. Figures show both unfiltered and 11-point averaged data.

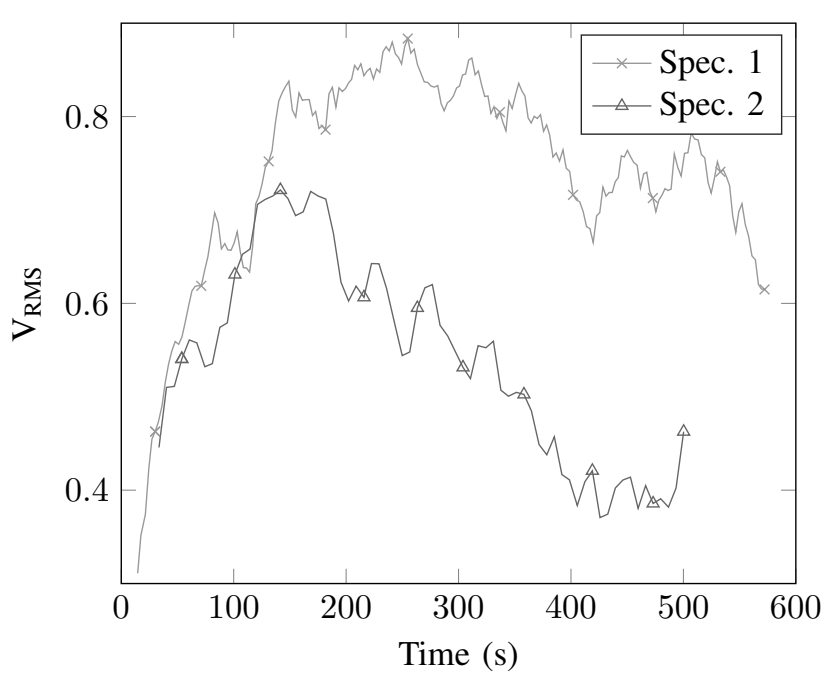

Fig. 7. RMS voltages, normalized and 11-point averaged, from measurements on two specimens applied with a $50 \mathrm{Nm}$ torque and rotated at $3000 \mathrm{rpm}$ until breakage.

\section{DISCUSSION AND CONCLUSIONS}

The similarities between the behaviour of the RMS voltages from our results and the results shown in Fig. 7 and Fig. 8 from [12] and Fig. 4 and Fig. 5 from [13] suggests that we do in fact measure the Barkhausen noise in the specimens. Comparing with the figures in [12], we see the same rapid increase and then the either fast or slow decrease of the signal. However, the signal in [12] returns to its starting level before plateauing for almost half of the test time. This can be attributed to a lower applied load and better enduring specimens. Similar behaviour is seen in the mechanical Barkhausen noise from [13]; although in that work the signal plateaus for a considerable time before failure of the specimen, a difference that can also be attributed to lower loading and more enduring specimens. The difference in behaviour between our work and the two referenced papers could also be attributed to the fatiguing of unknown magnitude and duration our specimens had been subjected to before our tests, which could have created an accelerated failure of the specimens.

For the conventional sensor, there are no typical or standard design, size or specifications [15]. Set-ups used by different authors and in different experiments vary with the shape of the yoke, number of turns of the excitation coil, shape of the pick-up coil, number of turns of the pick-up coil, strength of the excitation field, etc. Therefore a typical value of the power used for a conventional sensor cannot be found. However, a few examples from different authors can be recapitulated in order to get a general understanding of what is commonly used. White [16] uses a system where a maximum of $3.5 \mathrm{~W}$ of continuous power can be supplied to the inducing coil and does not state any numbers for signal amplification and data processing . Pal'a et al.'s [17] power supply feeds a maximum voltage of $30 \mathrm{~V}$ and maximum current of $2 \mathrm{~A}$, thus consuming at most $60 \mathrm{~W}$. They also do not provide any numbers for 
amplification and data processing. To summarise, the power consumption of the conventional sensor seems to lie in the range of a few to tens of Watts, without taking into account the power required for amplification and other peripherals, which can be assumed to be in comparison negligible.

The sensor presented in this paper only requires a fraction of the power required for the above mentioned Barkhausen sensors. With the high requirement on low noise from the amplifiers, the power consumption of the amplification circuit becomes in the order of 1 to $10 \mathrm{~mW}$ [18]. This power consumption falls in the range of power generation of energy harvesters [19], [20]. This allows us envision a sensor solution that can be small enough to integrate in a bearing and that can, through energy harvesting, be powered to amplify and process the signal as well as being able to communicate the condition of the bearing.

In conclusion, the proposed passive Barkhausen sensor show promising results in inducing and measuring Barkhausen noise. Preliminary testing have shown that the RMS voltages of the measured signal changes while the specimen is being fatigued. The way the recorded signal changes with time is similar to what have been shown in other studies that uses a conventional method of measuring Barkhausen noise or are measuring the mechanical Barkhausen noise. It is also shown that the proposed passive sensor design consumes far less power than a conventional Barkhausen sensor.

The preliminary results presented here are promising and provides a platform for evolving the proposed technique into a practical tool for condition monitoring. In this future development process it is of interest to study the influence on the detected Barkhausen noise from the magnitude of the relative motion as well as studies of the signal penetration depth into the material. Also, we need to study how the change from an alternating magnetic field to a constant but rotating magnetic field affects the measurement and if the method change causes a remanent magnetism in the specimen that in turn would reduce the Barkhausen noise signal strength over time.

With a fully developed and integrated low power sensor technology the real-time Barkhausen information indicating fatigue or deterioration in a raceway or rolling element will be a useful tool for bearing diagnostics and prognostics. The proposed sensor technology could then enable a condition based maintenance system, where the bearing itself acts as a condition indicator.

\section{ACKNOWLEDGEMENTS}

The authors would like to thank SKF AB for their financial support and valuable discussions and Vinnova for their financial support.

\section{REFERENCES}

[1] A. K. S. Jardine, D. Lin, and D. Banjevic, "A review on machinery diagnostics and prognostics implementing condition-based maintenance," Mechanical Systems and Signal Processing, vol. 20, no. 7, pp. 1483$1510,2006$.

[2] K. F. Martin, "A review by discussion of condition monitoring and fault diagnosis in machine tools," International Journal of Machine Tools and Manufacture, vol. 34, no. 4, pp. 527-551, 1994.
[3] B. Raj, V. Moorthy, T. Jayakumar, and K. Bhanu Sankara Rao, “Assessment of microstructures and mechanical behaviour of metallic materials through non-destructive characterisation," International Materials Reviews, vol. 48, no. 5, pp. 273-325, 2003.

[4] A. M. Al-Ghamd and D. Mba, "A comparative experimental study on the use of acoustic emission and vibration analysis for bearing defect identification and estimation of defect size," Mechanical Systems and Signal Processing, vol. 20, no. 7, pp. 1537-1571, 2006.

[5] T. Rocha, H. Ramos, A. L. Ribeiro, and D. Pasadas, "Sub-surface defect detection with motion induced eddy currents in aluminium," in Instrumentation and Measurement Technology Conference (I2MTC), 2015 IEEE International, 2015, pp. 930-934.

[6] S. Desvaux, M. Duquennoy, J. Gualandri, and M. Ourak, "The evaluation of surface residual stress in aeronautic bearings using the barkhausen noise effect," NDT \& E International, vol. 37, no. 1, pp. 9-17, 12004.

[7] B. D. Cullity and C. D. Graham, Introduction to magnetic materials, 2nd ed. Hoboken, N.J.: IEEE Press/Wiley, 2009.

[8] C. Gatelier-Rothea, J. Chicois, R. Fougeres, and P. Fleischmann, "Characterization of pure iron and (130 p.p.m.) carbon-iron binary alloy by barkhausen noise measurements: Study of the influence of stress and microstructure," Acta Materialia, vol. 46, no. 14, pp. 4873-4882, 1998.

[9] K. Tiitto, "Use of barkhausen noise in fatigue," Nondestructive Testing and Evaluation, vol. 5, no. 1, pp. 27-37, 1989.

[10] O. Stupakov, J. Pal'a, T. Takagi, and T. Uchimoto, "Governing conditions of repeatable barkhausen noise response," Journal of Magnetism and Magnetic Materials, vol. 321, no. 18, pp. 2956-2962, 2009.

[11] D. Jiles, Introduction to magnetism and magnetic materials, 1st ed. London: Chapman and Hall, 1991.

[12] E. S. Palma, T. R. Mansur, S. F. Silva, and A. Alvarenga, "Fatigue damage assessment in AISI 8620 steel using Barkhausen noise," International Journal of Fatigue, vol. 27, no. 6, pp. 659-665, 2005.

[13] M. Soultan, X. Kleber, J. Chicois, and A. Vincent, "Mechanical barkhausen noise during fatigue of iron," NDT \& E International, vol. 39, no. 6, pp. 493-498, 2006.

[14] B. Augustyniak, "Magnetomechanical damping evaluation from mechanical barkhausen effect," Materials Science Forum, vol. 119-121, pp. 559-564, 1993.

[15] G. Durin and S. Zapperi, "The barkhausen effect," in The Science of Hysteresis. Elsevier, 2006, vol. 2, pp. 181-267.

[16] S. A. White, "A Barkhausen noise testing system for CANDU feeder pipes," Ph.D. dissertation, Queen's University, Kingston, Canada, 2009. [Online]. Available: http://hdl.handle.net/1974/1994

[17] J. Pal'a, J. Bydžovský, and P. Švec, "Influence of magnetizing frequency and construction of pick-up coil on barkhausen noise," Journal of Electrical Engineering, vol. 55, no. 10/S, pp. 38-40, 2004.

[18] J. Delsing, J. Borg, and J. Johansson, "New Architecture for Efficient Data Sampling in Wireless Sensor Network Devices," in Proc. SENSORCOMM 2013, The Seventh International Conference on Sensor Technologies and Applications, 2013, pp. 90-96.

[19] F. Häggström, J. Gustafsson, and J. Delsing, "Energy harvesting technologies for wireless sensors in rotating environments," in Emerging Technology and Factory Automation (ETFA), 2014 IEEE, 2014.

[20] A. Harb, "Energy harvesting: State-of-the-art," Renewable Energy, vol. 36, no. 10, pp. 2641-2654, 2011. 